PROCEEDINGS OF THE

AMERICAN MATHEMATICAL SOCIETY

Volume 138, Number 12, December 2010, Pages 4479-4486

S 0002-9939(2010)10514-2

Article electronically published on July 22, 2010

\title{
LOWER VOLUME ESTIMATES AND SOBOLEV INEQUALITIES
}

\author{
STEFANO PIGOLA AND GIONA VERONELLI
}

(Communicated by Michael Wolf)

\begin{abstract}
We consider complete manifolds with asymptotically non-negative curvature which enjoy a Euclidean-type Sobolev inequality and we get an explicit lower control on the volume of geodesic balls. In case the amount of negative curvature is small and the Sobolev constant is almost optimal, we deduce that the manifold is diffeomorphic to Euclidean space. This extends previous results by M. Ledoux and C. Xia.
\end{abstract}

\section{INTRODUCTION}

A Riemannian manifold $(M,\langle\rangle$,$) of dimension \operatorname{dim} M=m>p \geq 1$ is said to support a Euclidean-type Sobolev inequality if there exists a constant $C_{M}>0$ such that, for every $u \in C_{c}^{\infty}(M)$,

$$
\left(\int_{M}|u|^{p^{*}} d \mathrm{vol}\right)^{\frac{1}{p^{*}}} \leq C_{M}\left(\int_{M}|\nabla u|^{p} d \mathrm{vol}\right)^{\frac{1}{p}},
$$

where

$$
p^{*}=\frac{m p}{m-p}
$$

and $d$ vol denotes the Riemannian measure of $M$. Clearly, (11) implies that there exists a continuous imbedding $W^{1, p}(M) \hookrightarrow L^{p^{*}}(M)$ and can be expressed in the equivalent form

$$
C_{M}^{-p} \leq \inf _{u \in \Lambda} \int_{M}|\nabla u|^{p} d \mathrm{vol},
$$

where

$$
\Lambda=\left\{u \in L^{p^{*}}(M):|\nabla u| \in L^{p} \text { and } \int_{M}|u|^{p^{*}} d \operatorname{vol}_{M}=1\right\} .
$$

The validity of (10), as well as the best value of the Sobolev constant $C_{M}$, have intriguing and deep connections with the geometry of the underlying manifold, many of which are discussed in the excellent lecture notes [5]. See also [8] for a survey in the more abstract perspective of Markov diffusion processes, and [9] for the relevance of (1) in the $L^{p, q}$-cohomology theory. For instance, we note that a complete manifold with non-negative Ricci curvature (but, in fact, a certain amount of negative curvature is allowed) and supporting a Euclidean-type Sobolev inequality is necessarily connected at infinity. This fact can be proved using (nonlinear) potential-theoretic arguments; see [10, [11.

Received by the editors March 12, 2010.

2010 Mathematics Subject Classification. Primary 53C21; Secondary 46 E35.

(C)2010 American Mathematical Society Reverts to public domain 28 years from publication 
It is known (see e.g. Proposition 4.2 in [5]) that

$$
C_{M} \geq K(m, p)
$$

where $K(m, p)$ is the best constant in the corresponding Sobolev inequality of $\mathbb{R}^{m}$. It was discovered by M. Ledoux, [7, that for complete manifolds of non-negative Ricci curvature, the equality in (2) forces $M$ to be isometric to $\mathbb{R}^{m}$. This important rigidity result has been generalized by $\mathrm{C}$. Xia, [13], by showing that, in case $C_{M}$ is sufficiently close to $K(m, p)$, then $M$ is diffeomorphic to $\mathbb{R}^{m}$. The key ingredient in the Ledoux-Xia argument is a sharp lower estimate for the growth of geodesic balls which depends explicitly on the Sobolev constant. Actually, it is known, 2, that the validity of (1) implies that there exists a (small) constant $\gamma=\gamma\left(m, p, C_{M}\right)>0$ (depending continuously on $C_{M}$ ) such that

$$
V\left(B_{t}\right) \geq \gamma V\left(\mathbb{B}_{t}\right) .
$$

However, to obtain the desired rigidity, one needs to get a sharp value for $\gamma$. In this paper, using a somewhat more geometric approach, we are able to extend this kind of estimate to manifolds with asymptotically non-negative curvature; see Theorem 1. As a consequence, using a theorem by S.-H. Zhu, [14, we will deduce rigidity even in this more general context.

Notation. In what follows, having fixed a reference origin $o \in M$, we set $r(x)=$ $\operatorname{dist}_{M}(x, o)$, and we denote by $B_{t}$ and $\partial B_{t}$ the geodesic ball and sphere of radius $t>0$ centered at $o$. The corresponding balls and spheres in the $m$-dimensional Euclidean space are denoted by $\mathbb{B}_{t}$ and $\partial \mathbb{B}_{t}$. Finally, the symbols $V\left(B_{t}\right)$ and $A\left(\partial B_{t}\right)$ stand, respectively, for the Riemannian volume of $B_{t}$ and the $(m-1)$ dimensional Hausdorff measure of $\partial B_{t}$.

Theorem 1. Let $(M,\langle\rangle$,$) be a complete, m-dimensional Riemannian manifold,$ $m \geq 3$, with

$$
{ }^{M} \operatorname{Ric}(x) \geq-(m-1) G(r(x)) \text { on } M
$$

for some non-negative function $G \in C^{0}([0,+\infty))$. Assume that $G$ satisfies the integrability condition

$$
\int_{0}^{\infty} t G(t) d t=b<+\infty
$$

and that the Euclidean-type Sobolev inequality (1) holds on $M$, for some $1<p<m$. Then

$$
e^{(m-1) b} V\left(\mathbb{B}_{t}\right) \geq V\left(B_{t}\right) \geq \hat{C}\left(m, p, C_{M}, b\right) V\left(\mathbb{B}_{t}\right)
$$

where

$$
\hat{C}\left(m, p, C_{M}, b\right)=e^{-b(m-1)}\left[\left(\frac{C_{M}}{K(m, p)}\right)^{p}+C_{M}^{p} C_{2}\right]^{-\frac{m}{p}}
$$

and

$$
0<C_{2}=C_{2}(m, p, b) \rightarrow 0, \text { as } b \rightarrow 0 .
$$

Using Theorem 1 we shall deduce the announced topological rigidity result.

Corollary 2. Given $m \geq 3, m>p$, there exist constants $b_{0}=b_{0}(m, p)>0$ and $\varepsilon_{0}=\varepsilon_{0}(m, p)>0$ such that the following holds: 
Let $M$ be an $m$-dimensional complete manifold supporting the Sobolev inequality (11) with $C_{M} \leq K(m, p)+\varepsilon$, for some $0<\varepsilon \leq \varepsilon_{0}$, and such that

$$
{ }^{M} \text { Sect } \geq-G(r(x)) \text { on } M \text {, }
$$

where $G$ satisfies (5) for some $0<b \leq b_{0}$. Then $M$ is diffeomorphic to $\mathbb{R}^{m}$.

\section{Proof of the Lower volume estimate}

Recall that, in $\mathbb{R}^{m}$, the equality in (1) with the best constant $C_{\mathbb{R}^{m}}=K(m, p)$ is realized by the (radial) Bliss-Aubin-Talenti functions $\phi_{\lambda}(x)=\varphi_{\lambda}(|x|)$ for every $\lambda>0$, where $|x|$ is the Euclidean norm of $x$ and $\varphi_{\lambda}(t)$ are the real-valued functions defined as

$$
\varphi_{\lambda}(t)=\frac{\beta(m, p) \lambda^{\frac{m-p}{p^{2}}}}{\left(\lambda+t^{\frac{p}{p-1}}\right)^{\frac{m}{p}-1}} .
$$

If we choose $\beta(m, p)>0$ such that

$$
\int_{\mathbb{R}^{m}} \phi_{\lambda}^{p^{*}}(x) d x=1
$$

then

$$
K(m, p)^{-p}=\int_{\mathbb{R}^{m}}\left|\varphi_{\lambda}^{\prime}(|x|)\right|^{p} d x
$$

and, by the standard calculus of variations, the extremal functions $\phi_{\lambda}$ obey the (non-linear) Yamabe-type equation

$$
\mathbb{R}^{m} \Delta_{p} \phi_{\lambda}=-K(m, p)^{-p} \phi_{\lambda}^{p^{*}-1},
$$

where

$$
\Delta_{p} u=\operatorname{div}\left(|\nabla u|^{p-2} \nabla u\right)
$$

stands for the $p$-Laplacian of a given function $u$.

Define

$$
\hat{\phi}_{\lambda}: M \rightarrow \mathbb{R} \text { as } \hat{\phi}_{\lambda}(x):=\varphi_{\lambda}(r(x)) .
$$

If we were in the assumptions of Ledoux and Xia, namely, if the Ricci curvature was non-negative, the idea of our proof would be simply to apply the Karp version of Stokes' theorem, [6], to the vector field $X_{\lambda}:=\hat{\phi}_{\lambda}\left|\nabla \phi_{\lambda}\right|^{p-2} \nabla \hat{\phi}_{\lambda}$, once we have observed that, by (9) and the Laplacian comparison theorem, each function $\hat{\phi}_{\lambda}$ on $M$ satisfies

$$
\Delta_{p} \hat{\phi}_{\lambda} \geq-K(m, p)^{-p} \hat{\phi}_{\lambda}^{p^{*}-1} .
$$

This would lead directly to inequality (2.2) in [13], and the desired volume conclusion can be deduced. The strategy for the proof of the general case stated in Theorem 1 is completely similar. Clearly, this time we have to take into account the (small) perturbations of (10) introduced by the negative curvature. Note also that, due to the possible presence of cut-points, all the computations have to be performed in the sense of distributions.

Proof of Theorem 1. Let $h \in C^{2}([0,+\infty))$ be the solution of the problem

$$
\left\{\begin{array}{l}
h^{\prime \prime}(t)-G(t) h(t)=0, \\
h(0)=0, h^{\prime}(0)=1,
\end{array}\right.
$$


and consider the $m$-dimensional model manifold $M_{h}$ defined as $M_{h}:=\left(\mathbb{R} \times \mathbb{S}^{m-1}\right.$, $\left.d s^{2}+h^{2}(s) d \theta^{2}\right)$, where $d \theta^{2}$ is the standard metric on $\mathbb{S}^{m-1}$. We shall use an index $h$ to denote objects and quantities referred to $M_{h}$. Thus, we denote by $\mathbb{B}_{t}^{h}$ and $\partial \mathbb{B}_{t}^{h}$ the geodesic ball and sphere of radius $t>0$ in $M_{h}$. Moreover, we introduce the family of functions $\phi_{\lambda, h}: M_{h} \rightarrow \mathbb{R}$ defined by $\phi_{\lambda, h}((s, \theta)):=\varphi_{\lambda}(s)$. For later purposes, we recall that $([14$, [10])

$$
\mathrm{V}\left(\mathbb{B}_{t}^{h}\right) \geq \mathrm{V}\left(\mathbb{B}_{t}\right), t \geq 0 .
$$

Furthermore, we observe that, according to the Bishop-Gromov comparison theorem and its generalizations, [3], [10], $A\left(\partial B_{t}\right) / A\left(\partial \mathbb{B}_{t}^{h}\right)$ is a decreasing function of $t>0$ and the following relations hold:

$$
\begin{aligned}
& A\left(\partial B_{t}\right) \leq A\left(\partial \mathbb{B}_{t}^{h}\right) \leq e^{b(m-1)} A\left(\partial \mathbb{B}_{t}\right), \\
& V\left(B_{t}\right) \leq V\left(\mathbb{B}_{t}^{h}\right) \leq e^{b(m-1)} V\left(\mathbb{B}_{t}\right)
\end{aligned}
$$

By the co-area formula, these imply that

$$
\begin{cases}\text { (i) } & \int_{M} \hat{\phi}_{\lambda}^{p^{*}} d \mathrm{vol} \leq \int_{M_{h}} \phi_{\lambda, h}^{p^{*}} d \operatorname{vol}_{h} \leq e^{b(m-1)}, \\ \text { (ii) } & \left|\nabla \hat{\phi}_{\lambda}\right| \in L^{p}(M), \\ \text { (iii) } & r(x)^{-1} \widehat{\phi}_{\lambda}\left|\nabla \widehat{\phi}_{\lambda}\right|^{p-1} \in L^{1}(M), \\ \text { (iv) } & \int_{B_{R}} \widehat{\phi}_{\lambda}\left|\nabla \widehat{\phi}_{\lambda}\right|^{p-1} d \mathrm{vol}=o(R) \text {, as } R \rightarrow+\infty .\end{cases}
$$

Here $d \mathrm{vol}_{h}$ stands for the Riemannian measure on $M_{h}$.

Now, by Laplacian comparison, [10, assumption (41) yields

$$
\Delta r \leq \frac{(m-1) e^{b}}{r}
$$

pointwise on $M \backslash \operatorname{cut}(o)$ and weakly on all of $M$. This means that

$$
-\int_{M}\langle\nabla r, \nabla \eta\rangle d \mathrm{vol} \leq \int_{M} \eta \frac{(m-1) e^{b}}{r} d \mathrm{vol}
$$

for all $0 \leq \eta \in W_{c}^{1,2}(M)$. Let $0 \leq \xi \in C_{c}^{\infty}(M)$ to be chosen later and apply (14) with

$$
\eta=-\left(\xi \hat{\phi}_{\lambda}\right)\left|\varphi_{\lambda}^{\prime}(r)\right|^{p-2} \varphi_{\lambda}^{\prime}(r)
$$

thus obtaining

$$
\begin{aligned}
& \int_{M} \varphi_{\lambda}^{\prime}(r)\left|\varphi_{\lambda}^{\prime}(r)\right|^{p-2}\left\langle\nabla r, \nabla\left(\xi \hat{\phi}_{\lambda}\right)\right\rangle d \mathrm{vol} \\
& \leq-\int_{M}\left|\varphi_{\lambda}^{\prime}(r)\right|^{p-2}\left[(p-1) \varphi_{\lambda}^{\prime \prime}(r)+\frac{(m-1) e^{b}}{r} \varphi_{\lambda}^{\prime}(r)\right]\left(\xi \hat{\phi}_{\lambda}\right) d \mathrm{vol} .
\end{aligned}
$$

On the other hand, according to (9),

$$
\left|\varphi_{\lambda}^{\prime}(t)\right|^{p-2}\left\{(p-1) \varphi_{\lambda}^{\prime \prime}(t)+\frac{m-1}{t} \varphi_{\lambda}^{\prime}(t)\right\}=-K(m, p)^{-p} \varphi_{\lambda}^{p^{*}-1}(t)
$$


for all $t>0$, and inserting into (15) gives

$$
\begin{aligned}
& -\int_{M} K(m, p)^{-p} \xi \hat{\phi}_{\lambda}^{p^{*}} d \mathrm{vol}+\int_{M} \hat{\phi}_{\lambda} \varphi_{\lambda}^{\prime}(r)\left|\varphi_{\lambda}^{\prime}(r)\right|^{p-2} \frac{(m-1)\left(e^{b}-1\right)}{r} \xi d \mathrm{vol} \\
& \leq-\int_{M} \xi\left|\nabla \hat{\phi}_{\lambda}\right|^{p} d \mathrm{vol}-\int_{M} \hat{\phi}_{\lambda} \varphi_{\lambda}^{\prime}(r)\left|\varphi_{\lambda}^{\prime}(r)\right|^{p-2}\langle\nabla r, \nabla \xi\rangle d \mathrm{vol} \\
& \leq-\int_{M} \xi\left|\nabla \hat{\phi}_{\lambda}\right|^{p} d \mathrm{vol}-\int_{M} \hat{\phi}_{\lambda} \varphi_{\lambda}^{\prime}(r)\left|\varphi_{\lambda}^{\prime}(r)\right|^{p-2}|\nabla \xi| d \mathrm{vol} .
\end{aligned}
$$

Now choose $\xi=\xi_{R}$ such that $\xi \equiv 1$ on $B_{R}, \xi \equiv 0$ on $M \backslash B_{2 R}$ and $|\nabla \xi|<2 / R$. Then, taking the limits as $R \rightarrow+\infty$ in (16) and recalling (13), we obtain

$$
\begin{aligned}
& \int_{M}\left|\nabla \hat{\phi}_{\lambda}\right|^{p} d \mathrm{vol}-K(m, p)^{-p} \int_{M} \hat{\phi}_{\lambda}^{p^{*}} d \mathrm{vol} \\
& \leq(m-1)\left(\frac{m-p}{p-1}\right)^{p-1} \beta^{p}\left(e^{b}-1\right) \lambda^{\frac{m-p}{p}} \int_{M}\left(\lambda+r^{\frac{p}{p-1}}\right)^{-(m-1)} d \mathrm{vol},
\end{aligned}
$$

proving that

$$
\frac{\int_{M}\left|\nabla \hat{\phi}_{\lambda}\right|^{p}}{\int_{M} \hat{\phi}_{\lambda}^{p^{*}}} \leq K(m, p)^{-p}+C_{1},
$$

where we have set

$$
C_{1}(m, p, \lambda, b):=(m-1)\left(\frac{m-p}{p-1}\right)^{p-1} \beta^{-\frac{p^{2}}{m-p}}\left(e^{b}-1\right) \frac{\int_{M}\left(\lambda+r^{\frac{p}{p-1}}\right)^{-(m-1)} d \mathrm{vol}}{\lambda \int_{M}\left(\lambda+r^{\frac{p}{p-1}}\right)^{-m} d \mathrm{vol}}
$$

By (12) and computing explicitly the integrals on $\mathbb{R}^{m}$, we get

$$
\begin{aligned}
\int_{M}\left(\lambda+r^{\frac{p}{p-1}}\right)^{-(m-1)} d \mathrm{vol} & \leq \int_{0}^{\infty} \frac{A\left(\partial \mathbb{B}_{t}\right) e^{b(m-1)}}{\left(\lambda+t^{\frac{p}{p-1}}\right)^{m-1}} \\
& =e^{b(m-1)} A\left(\partial \mathbb{B}_{1}\right) \lambda^{-\frac{m-p}{p}} \frac{\Gamma\left(m-\frac{m}{p}\right) \Gamma\left(\frac{m}{p}-1\right)}{\frac{p}{p-1} \Gamma(m-1)},
\end{aligned}
$$

where $\Gamma$ denotes the Euler Gamma function. On the other hand,

$$
\frac{V\left(B_{t}\right)}{\left(\lambda+t^{\frac{p}{p-1}}\right)^{m}} \leq \frac{V\left(\mathbb{B}_{t}^{h}\right)}{\left(\lambda+t^{\frac{p}{p-1}}\right)^{m}} \leq e^{b(m-1)} \frac{V\left(\mathbb{B}_{t}\right)}{\left(\lambda+t^{\frac{p}{p-1}}\right)^{m}} \rightarrow 0,
$$

as $t \rightarrow \infty$. Therefore, we can integrate by parts using the co-area formula, apply (3i), integrate by parts again and compute explicitly the integrals on $\mathbb{R}^{m}$, thus obtaining

$$
\begin{aligned}
\int_{M}\left(\lambda+r^{\frac{p}{p-1}}\right)^{-m} d \mathrm{vol} & =\int_{0}^{\infty} V\left(B_{t}\right)\left(-\frac{d}{d t}\left(\lambda+t^{\frac{p}{p-1}}\right)^{-m}\right) d t \\
& \geq \gamma \int_{0}^{\infty} V\left(\mathbb{B}_{t}\right)\left(-\frac{d}{d t}\left(\lambda+t^{\frac{p}{p-1}}\right)^{-m}\right) d t \\
& =\gamma \int_{0}^{\infty} A\left(\partial \mathbb{B}_{t}\right) \frac{d}{d t}\left(\lambda+t^{\frac{p}{p-1}}\right)^{-m} d t \\
& =\gamma A\left(\partial \mathbb{B}_{1}\right) \lambda^{-\frac{m}{p}} \frac{\Gamma\left(m-\frac{m}{p}\right) \Gamma\left(\frac{m}{p}\right)}{\frac{p}{p-1} \Gamma(m)} .
\end{aligned}
$$


Inserting into (18) and (17), it follows that

$$
\frac{\int_{M}\left|\nabla \hat{\phi}_{\lambda}\right|^{p}}{\int_{M} \hat{\phi}_{\lambda}^{p^{*}}} \leq K(m, p)^{-p}+C_{2},
$$

where

$$
C_{2}(m, p, b):=\frac{(m-1)^{2} p}{m-p}\left(\frac{m-p}{p-1}\right)^{p-1} \beta^{-\frac{p^{2}}{m-p}}\left(e^{b}-1\right) \frac{e^{b(m-1)}}{\gamma} .
$$

On the other hand, because of (13), we can use $\hat{\phi}_{\lambda}$ into (11) and get

$$
\frac{\int_{M}\left|\nabla \hat{\phi}_{\lambda}\right|^{p}}{\left(\int_{M} \hat{\phi}_{\lambda}^{p^{*}}\right)^{\frac{p}{p^{*}}}} \geq C_{M}^{-p}
$$

Combining (20) and (21) we obtain

$$
\int_{M} \hat{\phi}_{\lambda}^{p^{*}} \geq C_{3}^{-1}
$$

with

$$
C_{3}\left(m, p, b, C_{M}\right):=\left[\left(\frac{C_{M}}{K(m, p)}\right)^{p}+C_{M}^{p} C_{2}\right]^{m / p} .
$$

From this latter, using (13), (19), the co-area formula and integrating by parts, it follows that

$$
\begin{aligned}
0 & \leq C_{3} e^{b(m-1)} \int_{M} \hat{\phi}_{\lambda}^{p^{*}} d \mathrm{vol}-\int_{M_{h}} \phi_{\lambda, h}^{p^{*}} d \operatorname{vol}_{h} \\
& =\int_{0}^{\infty} v_{M, h}(t) V\left(\mathbb{B}_{t}^{h}\right) \frac{d}{d t}\left(-\varphi_{\lambda}^{p^{*}}(t)\right) d t,
\end{aligned}
$$

where, by Bishop-Gromov, the function

$$
v_{M, h}(t):=\left[C_{3} e^{b(m-1)} \frac{V\left(B_{t}\right)}{V\left(\mathbb{B}_{t}^{h}\right)}-1\right]
$$

is non-increasing. In view of (11), in order to prove (6), it is enough to show that $\lim _{t \rightarrow \infty} v_{M, h}(t) \geq 0$. By contradiction, suppose there exist positive constants $\epsilon$ and $T$ such that $v_{M, h}(t) \leq-\epsilon$ for all $t \geq T$. In this assumption, $T_{0}:=\sup \{t<T$ : $\left.v_{M, h}(t) \geq 0\right\}$ is well defined and $0<T_{0}<T$. Then

$$
\begin{aligned}
& \int_{0}^{\infty} v_{M, h}(t) V\left(\mathbb{B}_{t}^{h}\right) \frac{d}{d t}\left(-\varphi_{\lambda}^{p^{*}}(t)\right) d t \\
& \leq v_{M, h}(0) \int_{0}^{T_{0}} V\left(\mathbb{B}_{t}^{h}\right) \frac{d}{d t}\left(-\varphi_{\lambda}^{p^{*}}(t)\right) d t \\
& -\epsilon \int_{T}^{\infty} V\left(\mathbb{B}_{t}^{h}\right) \frac{d}{d t}\left(-\varphi_{\lambda}^{p^{*}}(t)\right) d t .
\end{aligned}
$$

Observe that the 1-parameter family of functions

$$
V\left(\mathbb{B}_{t}\right) \frac{d}{d t}\left(-\varphi_{\lambda}^{p^{*}}(t)\right)=\omega_{m} \frac{m p}{p-1} \beta(m, p)^{p^{*}} \lambda^{\frac{m}{p}} \frac{t^{\frac{1}{p-1}+m}}{\left(\lambda+t^{\frac{p}{p-1}}\right)^{m+1}}
$$


is decreasing in $\lambda$, provided $\lambda \gg 1$. Then we can apply the dominated convergence theorem to deduce

$$
\begin{aligned}
& \lim _{\lambda \rightarrow+\infty} \int_{0}^{T_{0}} V\left(\mathbb{B}_{t}^{h}\right) \frac{d}{d t}\left(-\varphi_{\lambda}^{p^{*}}(t)\right) d t \\
& \leq e^{b(m-1)} \lim _{\lambda \rightarrow+\infty} \int_{0}^{T_{0}} V\left(\mathbb{B}_{t}\right) \frac{d}{d t}\left(-\varphi_{\lambda}^{p^{*}}(t)\right) d t=0 .
\end{aligned}
$$

On the other hand, using (11) and the co-area formula, we have

$$
\begin{aligned}
\int_{0}^{\infty} V\left(\mathbb{B}_{t}^{h}\right) \frac{d}{d t}\left(-\varphi_{\lambda}^{p^{*}}(t)\right) d t & \geq \int_{0}^{\infty} V\left(\mathbb{B}_{t}\right) \frac{d}{d t}\left(-\varphi_{\lambda}^{p^{*}}(t)\right) d t \\
& \geq \int_{0}^{\infty} A\left(\partial \mathbb{B}_{t}\right) \varphi_{\lambda}^{p^{*}}(t) d t \\
& =\int_{\mathbb{R}^{m}} \phi_{\lambda}^{p^{*}}(x) d x=1,
\end{aligned}
$$

for all $\lambda>0$. Therefore, by (24),

$$
\lim _{\lambda \rightarrow+\infty} \int_{T}^{\infty} V\left(\mathbb{B}_{t}^{h}\right) \frac{d}{d t}\left(-\varphi_{\lambda}^{p^{*}}(t)\right) d t \geq 1
$$

Inserting (24) and (25) into (23) we conclude that, up to choosing $\lambda>0$ large enough,

$$
\int_{0}^{\infty} v_{M, h}(t) V\left(\mathbb{B}_{t}^{h}\right) \frac{d}{d t}\left(-\varphi_{\lambda}^{p^{*}}(t)\right) d t<0
$$

which contradicts (22). Setting $\hat{C}\left(m, p, C_{M}, b\right):=C_{3}^{-1} e^{-b(m-1)}$, we have thus proven the validity of ([6).

Corollary 2 now follows immediately from (6) recalling the explicit form of $\hat{C}$ given in (7) and using the next result due to S.-H. Zhu, 14, later quantified by M. Bazanfaré, [1].

Theorem 3. Let $(M,\langle\rangle$,$) be a complete m$-dimensional Riemannian manifold such that

$$
{ }^{M} \text { Sect } \geq-G(r(x)) \text { on } M,
$$

where $G$ satisfies (5) for some $b>0$. Then there exists an explicit constant $0<$ $\omega_{0}=\omega_{0}(b) \rightarrow 1 / 2$ as $b \rightarrow 0$ such that, if

$$
V\left(B_{r}(o)\right) \geq c V\left(\mathbb{B}_{r}\right), \forall r>0,
$$

and for some $c \geq \omega_{0}$, then $M$ is diffeomorphic to $\mathbb{R}^{m}$.

\section{REFERENCES}

[1] M. Bazanfaré, Open manifolds with asymptotically nonnegative curvature. Illinois J. Math. 49 (2005), 705-717. MR2210255 (2006m:53049)

[2] G. Carron, Inégalités isopérimétriques et inégalités de Faber-Krahn. Séminaire de Théorie Spectrale et Géométrie, No. 13, Année 1994-1995, 63-66. MR1715957

[3] I. Chavel, Riemannian Geometry: A modern introduction. Cambridge Studies in Mathematics 98. Cambridge University Press, 2006. MR2229062 (2006m:53002)

[4] J. Cheeger, T.H. Colding, On the structure of spaces with Ricci curvature bounded below. I. J. Differential Geom. 46 (1997), no. 3, 406-480. MR.1484888 (98k:53044)

[5] E. Hebey, Nonlinear Analysis on Manifolds: Sobolev Spaces and Inequalities. CIMS Lecture Notes (1999). Courant Institute of Mathematical Sciences. MR.1688256 (2000e:58011) 
[6] L. Karp, On Stokes' theorem for noncompact manifolds. Proc. Amer. Math. Soc. 82 (1981), 487-490. MR612746 (83g:58002)

[7] M. Ledoux, On manifolds with non-negative Ricci curvature and Sobolev inequalities. Comm. Anal. Geom. 7 (1999), 347-353. MR1685586 (2000c:53043)

[8] M. Ledoux, The geometry of Markov diffusion generators. Ann. Fac. Sci. Toulouse Math. (6) 9 (2000), 305-366. MR 1813804 (2002a:58045)

[9] V. Gol'dshtein, M. Troyanov, Sobolev inequalities for differential forms and $L_{q, p}$-cohomology. Jour. Geom. Anal. 16 (2006), 597-631. MR2271946 (2008a:58024)

[10] S. Pigola, M. Rigoli, A.G. Setti, Vanishing and finiteness results in geometric analysis. A generalization of the Bochner technique. Progress in Mathematics, 266. Birkhäuser Verlag, Basel, 2008. MR2401291 (2009m:58001)

[11] S. Pigola, A.G. Setti, M. Troyanov. The topology at infinity of a manifold supporting an $L^{p, q}-$ Sobolev inequality. Preprint.

[12] S. Pigola, G. Veronelli, An alternative proof of a rigidity theorem for the sharp Sobolev constant. arXiv:1002.0756v1

[13] C. Xia, Complete manifolds with nonnegative Ricci curvature and almost best Sobolev constant. Illinois J. Math. 45 (2001), 1253-1259. MR1894894 (2003h:53056)

[14] S.-H. Zhu, A volume comparison theorem for manifolds with asymptotically nonnegative curvature and its applications. Amer. J. Math. 116 (1994), 669-682. MR.1277451|(95c:53049)

Dipartimento di Fisica e Matematica, Università dell'Insubria - Como, via Valleggio 11, I-22100 Como, ItALy

E-mail address: stefano.pigola@uninsubria.it

Dipartimento di Matematica, Università degli Studi di Milano, via Saldini 50, I-20133 Milano, Italy

E-mail address: giona.veronelli@unimi.it 